\title{
Foreign Bodies in the Ear, Nose and Throat: An Experience in a Tertiary Care Hospital in Central Nepal
}

\author{
Ramesh Parajuli ${ }^{1}$ \\ ${ }^{1}$ Department of ENT, Head and Neck Surgery, Chitwan Medical \\ College Teaching Hospital, Bharatpur, Chitwan, Nepal \\ Int Arch Otorhinolaryngol 2015;19:121-123.
}

\begin{abstract}
Address for correspondence Ramesh Parajuli, Assistant Professor, Department of ENT and Head and Neck Surgery, Chitwan Medical College, Chitwan, Nepal, Chitwan 00977, Nepal (e-mail: drrameshparajuli@gmail.com).
\end{abstract}

\begin{abstract}
Introduction A foreign body $(\mathrm{FB})$ is an object or substance foreign to the location where it is found. FBs in the ear, nose, and throat are a common problem frequently encountered in both children and adults.

Objective To analyze FBs in terms of type, site, age, and gender distribution and method of removal.

Methods A retrospective study was performed in a tertiary care hospital in the central part of Nepal. The study period was from June 2013 to May 2014. The information was obtained from hospital record books.

Results A total of 134 patients had FBs in the ear, nose, or throat; 94 were males and 40 were females. Of the 134 patients, $70(52.23 \%)$ had FB in the ear, $28(20.89 \%)$ in the nose, and $36(26.86 \%)$ in the throat. The FB was animate (living) in $28(40 \%)$ patients with $\mathrm{FB}$ in the ear and $1(3.5 \%)$ patient with $\mathrm{FB}$ in the nose, but the $\mathrm{FB}$ was inanimate (nonliving) in any patient with $\mathrm{FB}$ in the throat, in $42(60 \%)$ patients with $\mathrm{FB}$ in the ear $\mathrm{FB}$,

Keywords

- foreign body

- ear

- nose

- throat

- endoscopy and in 27 (96.4\%) patients with FB of the nose. The FB was removed with or without local anaesthesia (LA) in $98(73.13 \%)$ patients, and only 36 patients $(26.86 \%)$ required general anaesthesia (GA). The most common age group affected was $<10$ years.

Conclusion FBs in the ear and nose were found more frequently in children, and the throat was the most common site of FB in adults and elderly people. Most of the FBs can be easily removed in emergency room or outpatient department.
\end{abstract}

\section{Introduction}

A foreign body (FB) is any object in a region it is not meant to be, where it can cause harm by its mere presence if immediate medical attention is not sought. ${ }^{1}$ It can be found in the ear, nose, and throat (ENT) region. FB may be classified as animate (living) and inanimate (nonliving). The inanimate FBs can further be classified as organic or inorganic and hygroscopic (hydrophilic) or nonhygroscopic (hydrophobic). ${ }^{2}$ The presence of FBs in the ENT region is one of the most common causes of otolaryngologic emergencies. FBs can be introduced spontaneously or accidently in both adults and children. Generally, FBs are more common in younger children; this may be due to various factors such as curiosity to explore orifices, imitation, boredom, playing, mental retardation, insanity, and attention deficit hyperactivity disorder, along with availability of the objects and absence of watchful caregivers. $^{3}$ The aim of this study is to analyze FBs in terms of type, site, age, and gender distribution and method of removal. received

August 23, 2014

accepted

November 5, 2014

published online

December 30, 2014
DOI http://dx.doi.org/

10.1055/s-0034-1397336. ISSN 1809-9777.
Copyright $\odot 2015$ by Thieme Publicações License terms Ltda, Rio de Janeiro, Brazil 


\section{Materials and Methods}

A retrospective study was performed in the Department of ENT, Head and Neck Surgery in a tertiary care hospital in the central part of Nepal. The study population includes the number of patients with ENT FB lodgment who presented in the Outpatient Department (OPD) or in the emergency room (ER) during the 1-year study period (June 2013 to May 2014). The data were obtained from the hospital record books. Anterior rhinoscopy and otoscopy examination were performed to diagnose FB of the nose and ear, respectively. Rigid or flexible nasal endoscopic examination was also performed in suspected cases of FB in the nasal cavity that was not visualized with anterior rhinoscopy. Similarly, examination under microscope was an additional method for diagnosis as well as useful for removal of FB of the ear. Instruments such as Jobson Horne probe, FB hook, Tilley forceps, and crocodile forceps were used in FB removal from the nose and ear. In addition to the previously mentioned instruments, syringing and suctioning were other methods for FB ear removal. Plain X-ray of the neck was done in patients with a history of FB ingestion. Flexible nasopharyngolaryngoscopy and flexible upper gastrointestinal endoscopy were done in cases where the FB was not visible in X-ray to rule out presence of an $\mathrm{FB}$ or to determine its site of impaction. This was followed by removal of the FB from the oropharynx/hypopharynx and esophagus with direct laryngoscopy or rigid esophagoscopy, respectively.

\section{Results}

During the study period, 134 patients visited this hospital with FB in the ENT area; 94 were males and 40 were females. Of the 134 patients, 70 (52.23\%) had FB in the ear, 28 (20.89\%) in the nose, and 36 (26.86\%) in the throat. The FB was removed with or without local anaesthesia (LA) in 98 (73.13\%) patients, and only 36 patients (26.86\%) required general anaesthesia (GA).

\section{Foreign Bodies in the Ear}

A total of 70 patients presented to the hospital with FB in the ear. Of these 70 patients, 28 (40\%) harbored animate (living) FBs. These were 22 cases of ticks, 4 cases of cockroach, 1 case each of ant and aural myiasis. The rest $(42 ; 60 \%)$ had inanimate (nonliving) FB in their ears. Of these 42 cases of nonliving FB, 15 were hygroscopic FB in the form of grams, peanuts, bean seed, and rice grain; the remaining 27 cases were of nonhygroscopic FB in the form of cotton, paper, eraser, broken matchstick/cotton bud, foam, and beads.

of the total 70 patients, $32(45.71 \%)$ were children $<10$ years of age.

Of 70 cases of FB in the ear, 66 were removed in the OPD and ER with or without LA and only 4 required examination under microscope under GA for removal of the FB; all of them were younger than 10 years of age.

\section{Foreign Bodies in the Nose}

Twenty-eight patients had FB lodged in the nose. Twentyseven patients (96.42\%), all of whom were children $<10$ years of age, had nonliving FB and only 1 patient had living FB (i.e., maggots), an adult patient with fungating growth due to carcinoma of maxilla. Of 27 cases with nonliving FBs, 10 patients had hygroscopic FB such as bean, peanut, corn, and grams, and 17 patients had nonhygroscopic FB such as eraser, paper, sponge, and plastic and metallic objects.

Of the total 28 patients with an FB in the nose, 27 (96.42\%) were of the age group $<10$ years. Most of the FBs were removed in the OPD and ER with the application of topical nasal decongestant. Only 2 patients required removal of the FB under GA.

\section{Foreign Bodies in the Throat}

A total of 36 patients presented with the complaint of ingestion of FB. The most common type of FB was meat bone/bolus in the form of chicken, mutton, or buffalo meat and the most common site of the impaction was cricopharyngeal junction in 21 patients (58.3\%). The other sites of FB impaction were oral cavity, oropharynx, hypopharynx, and thoracic esophagus.

All the ingested FB were inanimate, with 26 (72.22\%) being organic and 10 (27.77\%) being inorganic. Organic FBs were meat bolus and bone (fish, chicken, mutton, and buffalo meat). The inorganic FBs included denture, coin, and plastic and metallic objects.

Age of 60 or more years was the most common group involved with FB in the throat, with 10 patients presenting with FB impaction. Among all the patients who ingested an $\mathrm{FB}, 30$ patients (83.33\%) required GA under GA for FB removal.

\section{Method of FB Removal}

Removal of FB is not always easy. It requires proper instruments and skill. In our study, most of the nasal and aural FBs were removed in the ER or OPD with or without LA. Out of 134 FBs in the ENT, 36 (26.47\%) required GA for FB removal and rest $(98 ; 73.13 \%)$ were removed with or without LA.

\section{Discussion}

Adults and older children usually give a history of FB lodgment in ENT. But younger children are brought to the clinic by anxious parents or relatives. FBs may vary widely in shape, size, and composition, and the symptoms may range from asymptomatic to acute life threatening condition.

In our study, the most common age group affected was age $<10$ years, similar to results found in many other studies. $^{4-8}$ This may be due to the tendency of young children to lodge objects into the natural orifices of body, accidentally or intentionally.

The ear was the most common site for FBs in young children, who not only insert objects in their ears but also into the ears of their siblings and friends. Common ear FBs include cotton wool, bean, bead, paper/plastic, eraser, insect, paddy seed, and popcorn kernel. Patients usually present with earache, aural fullness, or ear discharge. Occasionally it may be asymptomatic and found incidentally during routine otoscopic examination. A high incidence of living FBs (i.e., ticks) in our study is explained by the fact that the people in 
the villages of Chitwan go to the jungle to collect fodder and graze cattle. Many people get ticks in the ear when they go for jungle safari, as Chitwan is a famous tourist destination in our country and attracts both domestic and international tourists. Examination under a microscope helps to confirm the presence of $\mathrm{FB}$ in the ear and aids in its removal under intravenous sedation/GA to minimize trauma to the tympanic membrane and external auditory canal. It is useful especially in children who are not cooperative to allow proper otoscopic examination when there is associated otitis externa. Ear syringing led to successful removal of most of the nonhygroscopic FBs. Negative pressure suctioning can be useful especially when there is aural or nasal discharge along with the FB.

Our study showed that there is predominance of FBs in the nose in younger children, which was seen in many other studies. ${ }^{1,3,7}$ Unilateral, foul-smelling, purulent nasal discharge in children must be regarded as due to FB until proved otherwise. With growth and cognitive development, the introduction of FB in the nostrils diminishes significantly, which is found only in patients with psychiatric disorders. FB in the nose or ear is usually unilateral, although it can be bilateral, as in one of our patient with FB grams in both nasal cavities and another case of FB erasers in both the external auditory canals. Sometimes there can be multiple ear or nose FBs as well.

FB ingestion is a common problem. The most frequently swallowed FBs in children include coins and metallic FBs (parts of playing objects), and meat bones (chicken bone/fish bone/mutton/buffalo meat) are common in adults and elderly patients. ${ }^{8,9}$ In our study, meat bone/bolus was the most common FB found inside the throat, and the most common site of lodgment was the cricopharyngeal region. Such patients come in clusters, especially during religious festivals like Dashain and Tihar. Heavy consumption of alcohol and eating meat simultaneously, especially during festivals, along with poor mastication may be the cause for meat bone/bolus impaction in adults. In elderly, edentulous patients, defective peristalsis due to age-related neuromuscular incoordination and poor masticating habits are the predisposing factors for the cause of impaction of meat bone/bolus in the esophagus. Moreover, in elderly people there are commonly other underlying pathologies that cause narrowing of the digestive tract. Coin was the most common FB in the throat in children in our study, which is similar to other studies ${ }^{7,9}$; this may be due to fact that the coins are often handed to younger children and they accidentally swallow because of their tendency to take things into the mouth, inadequate control of deglutition, and shouting or crying while playing or eating.
Plain X-ray of soft tissue in the neck is a cost-effective radiologic examination method useful in the evaluation of $\mathrm{FB}$ in the throat. We advise X-rays in patients with history of FB ingestion. Direct laryngoscopy was occasionally useful in the evaluation and removal of FB in the oropharynx and hypopharynx. FBs from the digestive tract are usually removed by rigid esophagoscopy. But flexible upper gastrointestinal endoscopy is useful especially in the case of ingestion of radiolucent FBs. Moreover, it helps to detect the site of impaction especially in patients with cervical spondylosis where neck extension is not possible and to remove the FBs, or to push the FB into the stomach (e.g., in cases of impacted meat bolus at the distal part of esophagus).

\section{Conclusion}

FBs in the ear and nose were found more frequently in children, and the throat was the most common site of FBs in adults and elderly people. Most of the nasal and aural FBs can be easily removed in the ER or OPD. Parents/caretaker should not allow children to play with coins or other small objects to prevent the risk of FB ingestion or insertion.

\section{References}

1 Sarkar S, Roychoudhury A, Roychaudhuri BK. Foreign bodies in ENT in a teaching hospital in Eastern India. Indian J Otolaryngol Head Neck Surg 2010;62(2):118-120

2 Carney AS, Patel N, Clarke R. Foreign bodies in the ear and the aerodigestive tract in children. In: Scott-Brown's Otorhinolaryngology, Head and Neck Surgery, 7th ed. London, UK: Edward Arnold; 2008:1184-1193

3 Shrestha I, Shrestha BL, Amatya RCM. Analysis of ear, nose and throat foreign bodies in Dhulikhel hospital. Kathmandu Univ Med J (KUMJ) 2012;10(38):4-8

4 Banerjee S. Concept of foreign body-its past and present. Indian J Otolaryngol Head Neck Surg 1999;51(1, Suppl 1):23-30

5 Das SK. Aetiological evaluation of foreign bodies in the ear and nose. J Laryngol Otol 1984;98(10):989-991

6 Higo R, Matsumoto Y, Ichimura K, Kaga K. Foreign bodies in the aerodigestive tract in pediatric patients. Auris Nasus Larynx 2003; 30(4):397-401

7 Ray R, Dutta M, Mukherjee M, Gayen GC. Foreign body in ear, nose and throat: experience in a tertiary hospital. Indian J Otolaryngol Head Neck Surg 2014;66(1):13-16

8 Adhikari P, Shrestha BL, Baskota DK, Sinha BK. Accidental foreign body ingestion: analysis of 163 cases. Int Arch Otorhinolaryngol 2007;11(3):267-270

9 Pokharel R, Adhikari P, Bhusal CL, Guragain RP. Oesophageal foreign bodies in children. JNMA J Nepal Med Assoc 2008; 47(172):186-188 